\title{
Women's Preference of Home Delivery in Wonago District, Gedeo Zone, Southern Ethiopia 2018
}

\author{
Abebe Alemu 1, *, Gedefa Amenu², Muhammed Feyiso ${ }^{3}$ \\ ${ }^{1}$ Department of Midwifery, College of Health Science and Medicine, Wachamo University, Hosana, Ethiopia \\ ${ }^{2}$ Department of Midwifery, College of Health Science and Medicine, Dilla University, Dilla, Ethiopia \\ ${ }^{3}$ School of Public Health, College of Health Science and Medicine, Dilla University, Dilla, Ethiopia \\ Email address: \\ aalemu72@yahoo.com (A. Alemu),dageamanu@gmail.com (G. Amenu), mamfys8@gmail.com (M. Feyiso) \\ ${ }^{*}$ Corresponding author
}

\section{To cite this article:}

Abebe Alemu, Gedefa Amenu, Muhammed Feyiso. Women's Preference of Home Delivery in Wonago District, Gedeo Zone, Southern Ethiopia 2018. Journal of Gynecology and Obstetrics. Vol. 7, No. 3, 2019, pp. 85-91. doi: 10.11648/j.jgo.20190703.16

Received: February 3, 2019; Accepted: May 31, 2019; Published: June 12, 2019

\begin{abstract}
Introduction: Maternal mortality and morbidity associated with pregnancy related obstetric complications is imposing substantial burden for developing countries. Maternal place of deliver and lack of skilled birth attendant during delivery period is the main determinant indicator for the maternal death. Majority of women of developing country do not decide or make choice on place of delivery before onset of labor. Thus, this study was aimed to assess maternal preference of place of delivery and associated factors among postnatal women in Wonaggo district, Gedeo Zone, Southern Ethiopia, 2018. Methods: Mixed study design; employing community-based cross-sectional study combined with in-depth interviews and focus group discussions was used. Multi-stage sampling technique was used to enroll 772 study participants. A pre-tested structured questionnaire was used to collect data. Data was entered to EpiInfo7 and exported to SPSS 20.0 for analysis. Descriptive and summary statistics were done. Logistic regression analysis was used to identify the association of different variables. Odds ratios and $95 \%$ Confidence interval were computed to determine the presence and strength of association. Results: according to this study finding, $25.6 \%$ of participants were preferred to deliver at their home. Maternal educational status ( $\mathrm{AOR}=2.1,95 \% \mathrm{CI}: 1.27-3.6)$, parity $(\mathrm{AOR}=1.5,95 \% \mathrm{CI}: 1.08,1.6)$, antenatal care follow up during pregnancy $(1.575$, $95 \%$ CI: $1.203,5.12)$, place of last delivery $(9.93,95 \% \mathrm{CI}: 2.355,4.192)$ and knowledge towards danger sign of obstetric complications were variables found to be significantly associated with maternal preference of place of delivery. In Conclusion, a significant proportion of respondents were preferred to deliver at home. Thus, improving women education status in the community, accessing quality antenatal care, providing quality obstetric care service and avoiding disrespect and abuse during child birth are toughly suggested directions in order to assure institutional delivery in Wonag Distric, Southern Ethiopia.
\end{abstract}

Keywords: Mothers, home delivery, Southern Ethiopia

\section{Introduction}

Globally, an estimated 289,000 women died annually due to obstetric emergencies. Pregnant women are at risk of unpredicted obstetric emergencies. Poor resource setting countries are the most burdened; especially sub-Saharan Africa and Asia are the most contributors of maternal mortality and morbidity which accounts for $99 \%$ of maternal death; sub-Saharan (62\%) and South Asia (24\%) together contribute $86 \%$ of the problem [1-3]. It has been reported that Ethiopia is one of the six countries that contributes about
$50 \%$ maternal death, the others being India, Nigeria, Pakistan, Afghanistan and the democratic republic of Congo [4]. Despite government commitment to millennium development goal five (MDG5) to reduce maternal death by three-quarters over the period of 1990 to 2015, maternal mortality ratio (MMR) still off track, estimated at 420 per 100,000 live births in Ethiopia [5].

The major interventions have been implemented to avert adverse pregnancy outcome are, preventing unplanned pregnancy, preventing or treating complications of pregnancies and averting death and disabilities from complications [6]. 
Studies revealed that the most contributing factor for maternal and child morbidity and mortality the place of delivery which is the most common challenge to give appropriate care during delivery. One in four babies worldwide is delivered without skilled birth attendants. A key strategy for reducing maternal morbidity and mortality is ensuring that every birth occurs with the assistance of skilled health personnel, meaning a medical doctor, nurse or midwife [7, 8].

Ethiopia is one the developing countries that adopted millennium development goal-5 to improve maternal health by three-fourth by the year of 2015 since 1990. Currently reports showed that there is significant improvement in maternal mortality showing decrement by 412/100, 000 live births. Although progress has been made, the target is not attained yet and not running on track of the target which needs more to work on $[9,10]$.

In Ethiopia, despite implementation has been done to improve maternal health, the use of reproductive health services remains low, and home delivery among women of child bearing age remains a public health problem. In Ethiopia number of birth attended by skilled birth attendant is $26 \%$, which is far from global report $[11,12]$. The level of utilization of unskilled attendants at birth appears unacceptability high and the majority of the mothers $(61 \%)$ still prefer to deliver at home. This was related to maternal awareness on informed choice and decision on place of delivery, lack of awareness on importance of institutional delivery, low quality antenatal care, socioeconomic factors, lack of transport facility, poor attitude towards health service provides, poor knowledge on onset their delivery, cultural and traditional factors are among the most listed factors by different scholars [12, 13].

In Ethiopia, maternal death due to obstetric complications during pregnancy, childbirth and postpartum period still substantial burden. Poor maternal health service utilization especially low choice of health institutional for delivery service utilization among pregnant mother before and at onset of delivery contributed to maternal mortality significantly. Although some studies have been done to address this area of concern in Ethiopia, no study was done to assess maternal choice of place of delivery and associated factors especially in the study area to identify the gaps, as far as my knowledge is concerned. The result of this study may provide insight information on women preference of place of delivery in the study area which could help in designing appropriate interventions and as a base for further wide scale studies. Thus, this study was aimed to assess maternal choice of place of delivery and associated factors among postnatal mothers of Wonado distric, Gedeo zone, Southern Ethiopia 2018.

\section{Method and Materials}

\subsection{Study Design and Period}

Mixed community-based cross-sectional study design, combining quantitative and qualitative data collection was conducted to assess maternal preference of place of delivery and associated factors among postnatal mothers of Wonago district from January 01 to March 302018.

\subsection{Sample Size Determination}

The sample size was determined using a single population proportion formula considering the following assumptions: $64.62 \%$ proportion of maternal preference of place of delivery, $5 \%$ margin of error. Multiplying by design effect, the final sample size was adjusted for a non- response rate of $10 \%$ and the total sample was 772 .

\subsection{Sampling Techniques}

Multistage sampling was used to reach study participants. Sample size was proportionally allotted to randomly selected kebeles in the District and a systematic sampling technique was used to enroll study participants.

\subsection{Data Collection and Analysis}

Data was collected through face to face interviews using a structured questionnaire. To control data quality training was given for data collectors, and questionnaires were cheeked for completeness a head of time data entry.

Data analysis was performed using SPSS version-20. Maternal preference place of delivery was calculated using frequency distribution and proportion of their preference was taken. Variables reaching a p-value of 0.2 on bivariate analysis were included in multiple logistic regression analysis and p-value $<0.05$ were declared as significantly associated with outcome variable. The degree of association between the independent and dependent variables was analyzed using odds ratios with $95 \%$ confidence intervals.

\subsection{Ethical Clearance}

Ethical clearance was obtained from the research ethical review committee of College of health science and medicine, Dilla University. A formal letter of support was sent to wonago district health Bureau by Dilla University and permission was obtained. Finally, written informed consent was obtained from each pregnant woman.

\section{Result}

\subsection{Socio-Demographic Characteristics}

A total of 769 study participants were responded making response rate of $99.6 \%$. About $609(79.2 \%)$ were rural in place of residence. Of the respondents three hundred twenty nine $(48.2 \%)$ were not attended school and husbands accounting for $320(41.7 \%)$ were not attended any formal school. Majority of study participants, 319 (41.5\%) were housewife and all of them were disclosed their monthly income and, 266 (34.6\%) were within income of 501-1000. About three hundred eighty seven (50.3\%) were decided their healthy service utilization by themselves (Table 1). 
Table 1. Socio-demographic characteristics of postnatal mothers of wonago district, Gedeo zone, southern Ethiopia, 2017 ( $n=769)$.

\begin{tabular}{lll}
\hline Variables category & Frequency & Percentage \\
\hline Age of the mother & & \\
\hline $15-19$ & 67 & 8.7 \\
$20-24$ & 224 & 29.1 \\
$25-29$ & 207 & 26.9 \\
$30-34$ & 184 & 23.9 \\
$>35$ & 87 & 11.3 \\
Religion affiliation & & \\
Protestant & 487 & 63.3 \\
Orthodox & 232 & 30.2 \\
Muslim & 47 & 6.1 \\
Others & 3 & 4 \\
Educational level of respondent & & \\
No schooling & 329 & 42.8 \\
Primary school & 166 & 21.6 \\
Secondary school & 142 & 18.5 \\
Above secondary school & 132 & 17.2 \\
Occupation & & \\
Housewife & 264 & 34.3 \\
Farmer & 291 & 37.8 \\
Trader & 97 & 12.6 \\
Government employ & 117 & 15.2 \\
Place of residence & & \\
Urban & 160 & 20.8 \\
Rural & 609 & 79.2 \\
Distance from home to health facility by car & & \\
less than one hour & 491 & 63.8 \\
greater than one hour & 278 & 36.2 \\
Family monthly Income & & \\
$<500$ & 101 & 13.1 \\
$501-1000$ & 266 & 34.6 \\
1001-1500 & 264 & 34.3 \\
$>1501$ & 138 & 17.9 \\
Decision makers on health service utilization & & \\
Self (mother) & 387 \\
Husband & 101 & 50.3 \\
Jointly & 281 & 13.1 \\
\hline & & 36.5 \\
\hline
\end{tabular}

\subsection{Obstetric Characteristics of Respondents}

Among the study participants, $648(84.3 \%)$ of the last pregnancies were intended. Six hundred seventy-nine $(88.3 \%)$ of respondents had at least antenatal follow up, but 377 $(55.2 \%)$ attended greater than equal to four antenatal care visits during last pregnancy. Five hundred twenty (67.6\%) of the study participants were give birth at health Institutions, and $436(84.2 \%)$ was spontaneous vaginal delivery. Among the respondents, 145 (18.9\%) has been experienced obstetric complications; for which obstructed labor/prolonged labor, $45(36.6 \%)$ take over the majority; followed by hemorrhage $33(26.8 \%)$ (Table 2).

Table 2. Obstetric characteristics of postnatal mothers, Wonago district, Gedeo zone, southern Ethiopia, 2017 (N=769).

\begin{tabular}{lll}
\hline Variables category & Frequency & Percentage \\
\hline Gravidity & & \\
\hline One & 94 & 12.2 \\
\hline
\end{tabular}

\begin{tabular}{lll}
\hline Variables category & Frequency & Percentage \\
\hline Gravidity & & \\
\hline Two-four & 445 & 57.9 \\
Five and above & 230 & 29.9 \\
Parity & & \\
One & 88 & 11.4 \\
Two-four & 474 & 61.6 \\
Five and above & 207 & 26.9 \\
Last pregnancy intended & & \\
Intended & 648 & 84.3 \\
Unintended & 121 & 15.7 \\
ANC last pregnancy & & \\
Yes & 679 & 88.3 \\
No & 90 & 11.7 \\
Gestational age at onset of ANC & & \\
$<16$ weks & 188 & 27.5 \\
17-28 wks & 335 & 49.0 \\
$>28$ wks & 160 & 23.4 \\
Place of last delivery & & \\
Home & 249 & 32.4 \\
Health institution & 520 & 67.6 \\
Route of institutional delivery & & 84.2 \\
Normal vaginally & 436 & 12.2 \\
Vaginal assisted & 63 & 3.7 \\
Cesarean section & 19 & 13.9 \\
Experience Complications & & 36.2 \\
Yes & 145 & \\
No & 624 & \\
Self (mother alone) & 386 & \\
Husband (alone) & 105 & \\
Jointly & 278 & \\
\hline & & \\
\hline & & \\
\hline & & \\
\hline
\end{tabular}

\subsection{Preference of Place of Delivery}

The magnitude of the respondent's preference to deliver at home was 195 (25.6\%) and the major reason for preferring home delivery was disrespect and abuse during child birth at health Institutions (figure 1).

\subsection{Factors Associated with Maternal Preference of Place of Delivery}

In bivariate analysis all the variables with a p-value of $<0.05$ were candidates for final multivariate logistic regression analysis.

On multivariate logistic regression analysis, maternal preference of place delivery was significantly associated with maternal educational status, antenatal follow up, place of last delivery and knowledge of obstetric complications. Respondents who attended college/ university were two times more prefer to delivery at health institution than mothers who didn't schooling at all (AOR=1.9; 95\%CI: 1.13-3.25). Mothers who had antenatal follow up during their last pregnancy were three times more likely prefer to delivery at health institution than home delivery than who didn't attend antenatal care follow up $(\mathrm{AOR}=3.24 ; 95 \% \mathrm{CI}$ : 2.9-8.2). In case of place of last delivery; those who were gave birth their recent infant at health institution were nine times more likely prefer to deliver their next child at health institution than who were delivered their neonate at home (9.3; 95\%CI: 2.2-3.8) (Table 3). 


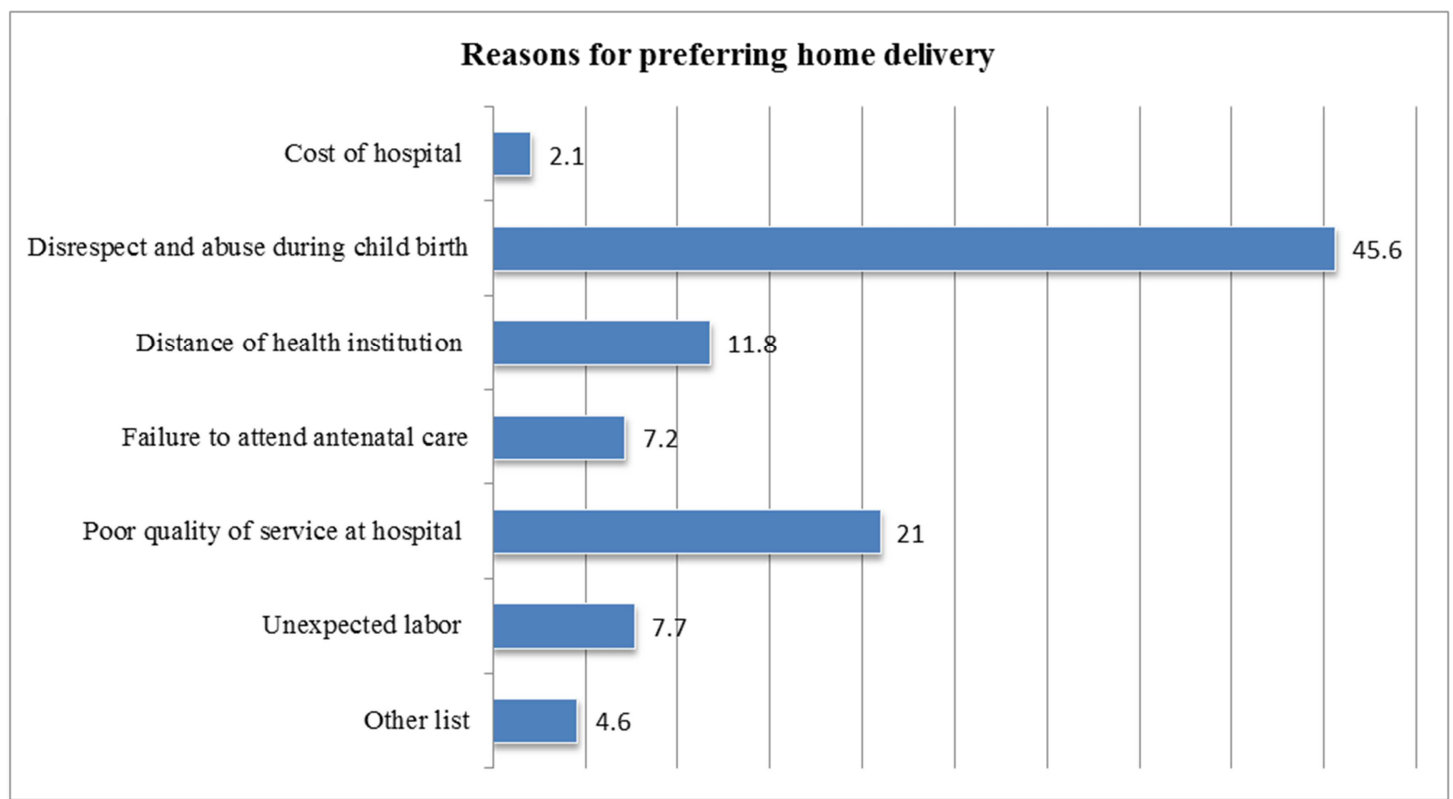

Figure 1. Percentage of the respondents with specific reason for preferring home delivery Wonago District, Gedeo zone, southern Ethiopia. (n=195).

Table 3. Multivariable analysis of factors associated with maternal preference of place of delivery among postnatal mothers of wonago district, Gedeo zone, Southern Ethiopia, 2017 ( $n=769)$.

\begin{tabular}{|c|c|c|c|c|}
\hline \multirow{2}{*}{ Variables } & \multicolumn{2}{|c|}{ Preference of place of delivery } & \multirow{2}{*}{ COR $(95 \%$ CI) } & \multirow{2}{*}{ AOR (95\%CI) } \\
\hline & Institution & Home & & \\
\hline \multicolumn{5}{|l|}{ Educational status } \\
\hline No schooling & $105(31.9 \%)$ & $224(68.1 \%)$ & Ref & Ref \\
\hline Primary school & $38(22.9 \%)$ & $128(77.1 \%)$ & $1.579(1.027,2.427)$ & $1.2(0.981,2.36)$ \\
\hline Secondary school & $31(21.8 \%)$ & $111(78.2 \%)$ & $1.678(1.059,2.688)$ & $1.502(0.94,2.4)$ \\
\hline College/ university & $23(17.4 \%)$ & $109(82.6 \%)$ & $2.221(1.339,3.684)$ & $2.150(1.28,3.61)$ \\
\hline \multicolumn{5}{|l|}{ Parity } \\
\hline One & $19(21.6 \%)$ & $69(78.4 \%)$ & Ref & \\
\hline Two-four & $110(23.2 \%)$ & $364(76.8 \%)$ & $1.619(0.990,3.188)$ & $1.3(0.69,2.18)$ \\
\hline Five and above & $68(32.9 \%)$ & $139(67.1 \%)$ & $1.777(1.129,2.320)$ & $1.5(1.08,1.64)$ \\
\hline \multicolumn{5}{|l|}{ Antenatal care } \\
\hline Yes & $519(76.4 \%)$ & $160(23.6 \%)$ & $2.265(1.436,3.572)$ & $1.6(1.20,5.12)$ \\
\hline No & $53(58.9 \%)$ & $37(41.1 \%)$ & Ref & Ref \\
\hline \multicolumn{5}{|l|}{ Place of last delivery } \\
\hline Health facilities & $418(80.4 \%)$ & $102(19.6 \%)$ & $11.84(2.258,3.872)$ & $9.9(2.35,4.19)$ \\
\hline Home & $185(74.4 \%)$ & $64(25.6 \%)$ & Ref & Ref \\
\hline \multicolumn{5}{|c|}{ Complication in last delivery } \\
\hline Yes & $45(31.00 \%)$ & $100(69.00 \%$ & $1.397(1.9,2.077)$ & $1.4(0.94,2.077)$ \\
\hline No & $152(24.4 \%)$ & $472(75.6 \%)$ & Ref & Ref \\
\hline \multicolumn{5}{|c|}{ Knowledge of obstetric danger sign } \\
\hline Yes & 333 & 95 & $2.471(1.559,3.577)$ & $2.1(1.534,3.3)$ \\
\hline No & 200 & 141 & Ref & \\
\hline
\end{tabular}

\subsection{Findings from Focus Group Discussions and In-Depth Interviews}

Five focus group discussions was done with the mothers who were gave birth within last one year and In-depth interview was done in collaboration health extension workers. FGD and in-depth interview was mainly addressed why they made choice towards place of last delivery at home and previous experiences with health facilities and the discussion points were themed (Table 4).

Table 4. Themes and sub-themes emanating from FGDs and in depth interviews about preference home delivery among postnatal mother in Wonago Distric, Southern Ethiopia.

\begin{tabular}{ll}
\hline Main themes & Sub-themes \\
\hline & No privacy at health institution \\
& Don't allow family to accompany mothers \\
Disrespect and abuse during child birth & Lack consent to do any procedure \\
& Redundant procedure at health institution \\
& Not respecting mothers and accompany \\
\hline
\end{tabular}




\begin{tabular}{ll}
\hline Main themes & Sub-themes \\
\hline \multirow{2}{*}{ Low awareness about health Institution delivery } & Believed skilled delivery is not necessary \\
& Low decision making power on their own health \\
Input factors & Additional expenses at health institution \\
Occurrence of delivery & Inadequate equipment and medication at health institution \\
\hline
\end{tabular}

Focus group discussion with mothers showed that; one of the reason that the women prefer home delivery over institution delivery was that; mothers get more support from her families appropriately at home than health institution. There were traditional birth attendants who come to the home to help the mother during onset of labor to attend the delivery at home.

"Women prefer home delivery than health institution. Because, those attendants allow the family to be with the mother and if they found out that the baby is not in the correct position; they will apply butter and massage over the abdomen to bring the baby to its normal position" said female participants of (FGD-1).

"I prefer to give birth at home than health institution because; I was delivered my first child at health institution before three years after I was referred to dilla hospital from health center after I stayed at health center for one day without any help.. When I was delivered at dilla hospital, I was in very severe pain. When I asked the doctor to allow my family to be with me he didn't allow my partners to enter delivery room. Unfortunately, he left the room and I gave birth without any support. I will never and ever forget that moment in my life. Therefore, I never want to go to hospital again" (said a 31 yrs. old mother from (FDG 2).

As women who preferred home delivery declared that some health professionals didn't maintain privacy during virginal examination, delivery and provide psychological support.

"From my previous experience, I am afraid of delivering in health institution because everybody in the delivery room watches our naked part. There is no covering during vaginal examination and all professionals crowded to see my body which made me nervous. They are not respecting us. So I won't have these problems if I give birth at my home by calling traditional birth attendants at my home. May be I go to hospital if I encounter difficulty which beyond my family at home" said participants from FDG2.

The main point rose from one focus discussion group, the majority of obstetric care providers were not well committed to evaluate, diagnosis and managed the problem timely as per its urgency.

"I gave a birth my recent child at my home. When I go to health center with complaint of sever pushing down pain; then health care provider examine and told me as I am not in labor and send be back to my home to come back after a week. But after I was reach my home labor pain was more increased progressively and I gave a birth my baby at my home on the same day. So I believe that she has no skill to examine and attend my labor. So need to go to health center for delivery" said the FGD.

Previous experience of home delivery also refrain mother from health institution delivery. Mothers continue to deliver at home if they had gave a birth their previous child without complication at home. Implies that mother prefer hospital delivery if they encounter complication from previous experience of home delivery.

"I was delivered three of my children at health center and the other one at my home. I had no problem related to delivery of some of my children, so I gave birth at home. But for the fourth baby, labor was last two day and also there was bleeding. Then I was referred to the hospital and delivery was happened at hospital by operation. During that time; I feel secured if I give birth at my home." (Said FGD4).

The other issue raised by the participants was about financial constraints during onset of labor. This is associated with accidental onset of labor and majority of the labor came during night during which going to health institution is impossible due to lack of transport at night. This shows than there is low birth preparedness and complication readiness awareness among the women.

A 23yrs old mother who was delivered her recent child at home explained the reason behind her decision to deliver at home: "I had antenatal follow up at health center three times. I was counseled on my pregnancy by health workers but my labor was started accidentally at night almost around 8:00 o'clock local time). Transportation is not available at night to go hospital for deliver and even since the labor was occurred accidentally we haven't prepared for the delivery. I gave a birth in late night without any complications" (FGD2)

\section{Discussion}

The finding of this study has shown that magnitude of maternal preference of place of delivery and associated factors among postnatal mothers in Wonago District Gedeo Zone, Southern Ethiopia 2017.

According to this study finding; the magnitude of maternal preference of home delivery was $197(25.6 \%)$ and 572 $(74.4 \%)$ respondents were chosen to delivery at health institution. The finding of this study is in line with study done in Debremarkos [22], but higher than study done finding in Jimma (64.62\%), and Haramaya (58.7\%) in Ethiopia $(20,28)$. This might be due to difference in participants of the study and time. The studies conducted at Jimma Zone and Haramaya were included the pregnant women, while in this study postnatal mothers who were gave birth within last one year were enrolled. The finding in this study was less when compared to study done in Benchi-Meji, Ethiopia and Tenzania [28, 29]. This might be due to different in between both country population and strength of 
the health service delivering in both Countries.

In this study, a higher percentage of women preferred Institutional delivery when compared to study conducted in Switzerland [31]. The difference could be mainly due to the difference in a model of delivery service. In some developed countries, delivery at home was attended by trained professionals who recognize complications and provider referral service. In developed countries, mostly home delivery were attended by skilled professionals, thus why the percentage of preference of Institutional delivery was low relatively in relation to this study outcome.

In this study, the result revealed that disrespect and abuse during child birth, lack of antenatal care follow up, lack of support to get health service, and unsatisfactory delivery services in the health facilities were found to be reason for preference of home delivery among postnatal mothers. These study finding is in line with other studies results in Ethiopia, Nigeria, Nepal, Kenya and Tanzania respectively [15, 18, 20, 22, 27-30, 32].

In this study, the result shown that maternal education was found to be significantly associated with the preference of the place of delivery. Those mothers who had attended college and University level education were two times more likely prefer to delivery in health institutions as compared to women who attended lower level education and not attended school. This finding is consistent with study conducted in the different parts of Ethiopia, Nigeria, Tanzania, Kenya, Nepal and Bangladesh [18, 20-22, 25, 27-30, 33, 34]. This might be due to the fact that, education is important to easily understand health message from different sources and also it enhances their autonomy to decide on their reproductive issue.

According to this study finding, parity was significantly associated the women preference of the place of birth. The multiparous women were more prefer institutional delivery than primparous women. This might be due to previous experiences of pregnancy and child birth, which may create opportunity for women to have health promotion. This finding was consistent with studies conducted in Haramaya, Jimma, Benchi-Maji and Nigeria [18, 28, 29, 35].

Antenatal follow up during pregnancy was significantly associated with maternal preference of place of delivery. Women's who had antenatal follow up were 1.5 times more likely preferred institutional delivery as compared to those who had no antenatal follow up. This finding is in line with study done in Nepal, Kenya, Ethiopia and Tanzania [18, 20]. This could be due to the fact that, antenatal care follow up provides an important opportunity for information, education and communication and also it allows the mothers to heard about obstetric danger signs during pregnancy.

Place of last delivery is also another factors strongly associated maternal preference of place of delivery. Mothers who were previously gave birth at health institution were about 9 times more likely prefer to deliver at health institution than and mothers who were previously delivered at home. This finding is in line with study done in BenchiMaji, Ethiopia and Kenya [21, 24, 29]. This might be due to the fact that, women who were gave birth at health institution might be aware of importance of institution delivery from service they were received and also counseled on future delivery place.

\section{Concussion}

The study finding shown that 195 (25.6\%) of mothers still prefer home as their place of delivery. Thus, improving women education, accessing quality antenatal care, providing quality obstetric care service and avoiding disrespect and abuse during child birth are toughly suggested directions in order to assure institutional delivery in Wonago district, Southern Ethiopia.

\section{Declaration}

\section{Competing Interests}

All authors declare that they have no competing interests

\section{Ethics Approval and Consent to Participate}

Ethical approval was gotten from Dilla University, College of Health Ethical committee which was dedicated to evaluating ethical consideration of researchers and informed written consent was obtained from study participants during data collections.

\section{Consent for Publish}

Not applicable

\section{Availability of Data and Materials}

All necessary data will be availed on request

\section{Funding}

Not applicable

\section{Clinical Trial Registry Number}

Not applicable

\section{Author's Contributions}

All authors equally contributed for this research work

\section{Acknowledgements}

We would like to thank study participants for their willingness to participate in this research work.

\section{References}

[1] Millennium T, Goals D. The Millennium Development Goals Report 2015. 2015;

[2] Report MDG. Assessing progress in africa toward the millennium development goals. 
[3] The Sustainable Development Goals of the Post-2015 Agenda: Comments on the OWG and SDSN Proposals. 2015; (February).

[4] Killen B, Maher D, Piva P, Richter-airijoki H, Toupin J, Travis P, et al. Health and the Millennium Development Goals. 2015;

[5] Millennium T, Goals D. The Millennium Development Goals Report 2014. 2014;

[6] Wilson W. Using evidence for policies to reduce maternal mortality • Role of evidence in maternal health. 2008;

[7] Millennium T, Goals D. The Millennium Development Goals Report 2013. 2013;

[8] Jun e 2015. 2015;

[9] Agency CS, Ababa A. Ethiopia Mini Demographic and Health Survey. 2014; (August).

[10] Are AD. ASSISTED Are we on the threshold of change? Towards a new Global Strategy for Women's, Children's and Adolescents' Health.

[11] Agency CS, Ababa A. Ethiopia Demographic and Health Survey. 2012; (March).

[12] Analysis I. Maternal Health in Ethiopia Trends in. 2012; (December).

[13] Tarekegn SM, Lieberman LS, Giedraitis V. Determinants of maternal health service utilization in Ethiopia: analysis of the 2011 Ethiopian Demographic and Health Survey. 2014; 1-13.

[14] Kabir MA. SAFE-DELIVERY PRACTICES IN RURAL BANGLADESH AND ITS ASSOCIATED FACTORS: EVIDENCE FROM BANGLADESH DEMOGRAPHIC AND HEALTH SURVEY-2004 Mohammad A. Kabir. 2007; 4 (2): $67-72$.

[15] Shrestha SK, Banu B, Khanom K, Ali L, Thapa N. Changing trends on the place of delivery: why do Nepali women give birth at home? 2012; $1-8$.

[16] Speizer IS, Story WT, Singh K. Factors associated with institutional delivery in Ghana: the role of decision-making autonomy and community norms. 2014; 1-13.

[17] Pfeiffer C, Mwaipopo R. Delivering at home or in a health facility? health-seeking behaviour of women and the role of traditional birth attendants in Tanzania. 2015;

[18] Publications MJ, Envuladu EA, Agbo HA, Lassa S, Kigbu JH, Zoakah AI. Factors determining the choice of a place of delivery among pregnant women in Russia village of Jos North, Nigeria : achieving the MDGs 4 and 5. 2013; 2 (1): 23-7.

[19] Wanjira C, Mwangi M, Mathenge E, Mbugua G, Ng Z. Delivery Practices and Associated Factors among Mothers Seeking Child Welfare Services in Selected Health Facilities in Nyandarua South District, Kenya. 2011.

[20] Zone J, West S, Study S, Yegezu RT, Kitila SB. Women 's Health Care Assessment of Factors Affecting Choice of Delivery Place among Pregnant. 2015; 4 (1): 1-4.

[21] Fira A, Ababulgu FA, Bekuma TT. Delivery Site Preferences and Associated Factors among Married Women of Child Bearing Age in Bench Maji Zone, Ethiopia. 2011; (8).

[22] Bayu H, Adefris M, Amano A, Abuhay M. Pregnant women's preference and factors associated with institutional delivery service utilization in Debra Markos Town, North West Ethiopia: A community based follow up study. BMC Pregnancy Childbirth. 2015; 15 (1).

[23] Fikre AA, Demissie M. Prevalence of institutional delivery and associated factors in Dodota Woreda (district), Oromia regional state, Ethiopia. 2012; 1-6.

[24] Kawakatsu Y, Sugishita T, Oruenjo K, Wakhule S, Kibosia K, Were E. Determinants of health facility utilization for childbirth in rural western Kenya : cross-sectional study. 2014.

[25] Shiferaw S, Spigt M, Godefrooij M, Melkamu Y, Tekie M. Why do women prefer home births in Ethiopia? 2013.

[26] Worku AG, Yalew AW, Afework MF. Factors affecting utilization of skilled maternal care in Northwest Ethiopia: a multilevel analysis. 2013.

[27] Journal GI. Factors Influencing Women 's Choice of Place of Delivery in Urban and Peri Urban Areas of Gondar Town, North West of Ethiopia. 2015; 2 (3): 2-5.

[28] Sciences M, Ababa A, Sciences M. THE PHARMA INNOVATION - JOURNAL Factors Affecting Choice of Delivery Place among Women in Haramaya Woreda, Oromia Regional State, Eastern. 2015; 2-9.

[29] Ababulgu FA, Bekuma TT. Delivery Site Preferences and Associated Factors among Married Women of Child Bearing Age in Bench Maji Zone, Ethiopia. Ethiop J Health Sci [Internet]. 2016; 26 (1): 45-54. Available from: http://www.pubmedcentral.nih.gov/articlerender.fcgi?artid=47 $62959 \&$ tool $=$ pmcentrez\&rendertype $=$ abstract

[30] Ngowi AF, Kamazima SR, Kibusi S, Gesase A, Bali T. Women's determinant factors for preferred place of delivery in Dodoma region Tanzania: A cross sectional study. Reprod Health. 2017; 14 (1): 1-8.

[31] Ackermann-liebrich U, Voegeli T, Giunter-witt K, Kunz I, Ziillig M. Home versus hospital deliveries : follow up study of matched pairs for procedures and outcome. 1996; 313 (November): 1313-8.

[32] Abeje G, Azage M, Setegn T. Factors associated with Institutional delivery service utilization among mothers in Bahir Dar City administration, Amhara region : a community based cross sectional study. 2014; 1-7.

[33] Belay AS, Sendo EG. Factors determining choice of delivery place among women of child bearing age in Dega Damot District, North West of Ethiopia: A community based cross- sectional study. BMC Pregnancy Childbirth [Internet]. 2016; 16 (1): 1-8. Available from: http://dx.doi.org/10.1186/s12884-016-1020-y

[34] Abdella M, Abraha A, Gebre A, Reddy PS. Magnitude and Associated Factors for Home Delivery Among Women Who Gave Birth in Last 12 Months in Ayssaita, Afar, Ethiopia2016. A Community Based Cross Sectional Study. Glob J Fertil Res. 2017; 2 (1): 30-9.

[35] Women J, Care H, Zone J, West S, Study S, Yegezu RT, et al. Journal of Women' $s$ Health Care Assessment of Factors Affecting Choice of Delivery Place among Pregnant. 2015; 4 (1): 1-4.

[36] Kruk ME, Paczkowski M, Mbaruku G, De Pinho H, Galea S. Women's preferences for place of delivery in rural Tanzania: A population-based discrete choice experiment. Am J Public Health. 2009; 99 (9): 1666-72. 